\title{
LAPATINIB-BASED THERAPY FOR WOMEN WITH ADVANCED/ METASTATIC HER2 POSITIVE BREAST CANCER
}

\author{
J. Zekri', 2, M. Mokhtar ${ }^{3}$, S.M. Karim ${ }^{1,2, *}$, T. Darwish', M. Al-Foheidi', \\ A. Rizvi ${ }^{1}$, S. Al-Rehaily ${ }^{4}$ M. Mahrous ${ }^{5}$, M. Mansour ${ }^{6}$ \\ ${ }^{1}$ Department of Oncology, King Faisal Specialist Hospital \& Research Centre, Jeddah 21499, Saudi Arabia \\ ${ }^{2}$ College of Medicine, Al-Faisal University, Riyadh 11533, Saudi Arabia \\ ${ }^{3}$ Department of Oncology, King Abdullah Medical City and Oncology Center, Makkah 21955, Saudi Arabia \\ ${ }^{4}$ Department of Oncology, National Guard Hospital, Jeddah 21423, Saudi Arabia \\ ${ }^{5}$ Department of Hematology and Oncology, King Fahad Hospital, Madinah 42351, Saudi Arabia \\ ${ }^{6}$ Department of Oncology, Erfan Hospital, Jeddah 23442, Saudi Arabia
}

\begin{abstract}
Background: Lapatinib alone or in combination with other agents, mostly capecitabine is used for patients with advanced/metastatic HER2 positive breast cancer $\left(\mathrm{HER2}^{+} \mathrm{BC}\right)$ after progression on trastuzumab based therapy. Here we report our experience with lapatinib based therapy in this setting. Material and Methods: 67 consecutive patients received lapatinib based therapy. $58(86.6 \%)$ received lapatinib + capecitabine (LC), $7(10.4 \%)$ with other agents and $2(3.0 \%)$ as single agent lapatinib. Data was collected from patients' records retrospectively. Results: Objective response to lapatinib based therapy in 64 evaluable patients was $\mathbf{6 4 . 0} \%$ in all patients and $\mathbf{6 4 . 0} \%$ in patients who received LC. Median progression free survival and overall survival were 10 and 27 months in all patients and 10 and 17 months in patients who received LC, respectively. $16(24.0 \%)$ patients had dose delay $>1$ week and/or dose reduction. Conclusion: Lapatinib based therapy is an effective treatment for women with advanced/metastatic HER2 ${ }^{+}$BC after prior exposure to trastuzumab. It yields meaningful response rates, progression free and overall survival. Some patients require dose adjustments.
\end{abstract}

Key Words: lapatinib-based therapy, metastatic breast cancer, HER2 positive breast cancer, trastuzumab.

The human epidermal growth factor receptor 2 (EGFR2 or HER2 or Erb-B2) is a member of the EGFR family of tyrosine kinase receptors. HER2 is a proto-oncogene located at the long arm of human chromosome 17 and is overexpressed in $15-30 \%$ of breast cancers (BC). It plays an important role in the development and progression of cancer and is strongly associated with disease recurrence and poor prognosis $[1,2]$. Clinical research over the recent 2 decades contributed successfully to the development drugs that specifically target HER2 receptors. Trastuzumab (T), a monoclonal antibody that binds to extracellular domain of the receptor improves the outcome of patients with HER2 positive breast cancer $\left(\mathrm{HER} 2^{+} \mathrm{BC}\right)$ and is licensed for the use in early and advanced/metastatic HER2 ${ }^{+}$breast cancer (A/ MHER2 $\left.{ }^{+} B C\right)[3,4]$. When tumour cells eventually develop resistance to T, further inhibition of HER2 receptor signaling is needed to overcome this escape. Lapatinib (L) is an orally active small molecule that inhibits the tyrosine kinases of HER2 and EGFR

Submitted: April 08, 2015.

*Correspondence: E-mail: skarim@kfshrc.edu.sa Fax: 00966126677777 (Ext 64030)

Abbreviations used: A/MHER2'BC - advanced/metastatic HER2 positive breast cancer; $\mathrm{BC}$ - breast cancer; $\mathrm{C}$ - capecitabine; EGFR2 - epidermal growth factor receptor 2; FDA - Food and Drug Administration; HER2 - human epidermal receptor 2; L - lapatinib; LBT - lapatinib-based therapy; LC - lapatinib plus capecitabine; LT - lapatinib plus trastuzumab; LV - lapatinib plus vinorelbine; OS - overall survival; PFS - progression free survival; RECIST - Response Evaluation Criteria in Solid Tumors; T - trastuzumab.
(HER1). In 2007, the American Food and Drug Administration (FDA) licensed $L$ for use in combination with capecitabine $(C)$ in the treatment of patients with $A$ / MHER2 ${ }^{+} B C$ who had received prior therapy including $T$. This approval was based on the results of a randomized phase III trial showing a longer time to progression in favor of the group receiving $L$ [5]. Subsequent clinical trials confirmed the efficacy of single agent $L$ and $L$ in combination with other anti-cancer agents including letrozole, anastrazole, $T$ and a number of other chemotherapeutic agents [6-9]. Most of the available research studied lapatinib-based therapy (LBT) in patients from western countries. However, LBT is used all over the world for women with A/MHER2 ${ }^{+} \mathrm{BC}$. There is scarcity of data on efficacy and tolerability in these women and certainly in women from Arabic ethnicity.

Here we retrospectively report our experience with LBT in women with $\mathrm{A} / \mathrm{MHER} 2^{+} \mathrm{BC}$ treated in 5 different oncology units in Western region of Saudi Arabia.

\section{MATERIALS AND METHODS}

All consecutive patients who started LBT for A/MHER2 ${ }^{+}$BC between February 2008 and June 2013 were eligible for inclusion in the study. LBT was identified as single agent $L$ or $L$ in combination with any anti-cancer hormonal therapy or chemotherapy. 72 patients met the eligibility criteria. However, the medical records of 5 patients were unobtainable or contained inadequate information. Adequate clinical data was available for 67 patients and are the subject of this report. $58 / 67(86.6 \%)$ received lapatinib plus capecitabine (LC). 
Starting dose of $L$ was $1250 \mathrm{mg}$ daily and of C $1000 \mathrm{mg} / \mathrm{m}^{2}$ twice a day on days $1-14$ every 3 weeks. Data was collected from patients' paper and electronic records retrospectively by the treating medical oncologists. Progression free survival (PFS) was defined as time from starting LBT until first evidence of radiological or obvious clinical progression or last follow up. Overall survival (OS) was defined as time from starting LBT until death or last follow up.

Radiological imaging and/or reports were reviewed by the treating medical oncologists to document response and progression. Central radiology review was not obtained. Response Evaluation Criteria in Solid Tumors (RECIST) were used to guide response assessment. During LBT, patient follow up generally consisted of regular physical examination and relevant hematological and biochemical laboratory assessment every 3 weeks. Radiological response assessment did not follow a predefined protocol. Instead it was carried out according to clinical impression and physicians' discretion. However, generally most patients had radiological assessment every $2-3$ months mostly by CT scan.

Due to retrospective nature of the study and concerns about incomplete documentation of toxicity data in patients' records, surrogates for toxicity and tolerability was used. These surrogates were dose delay for $>1$ week (including discontinuation) and dose reduction in any component of the regimen.

Statistical analysis was performed using SPSS 11.5 for Windows. Response rate was expressed a percentage of evaluable patients. PFS and OS were calculated using life tables and Kaplan - Meier estimator. Institutional review board approval was granted for this study.

\section{RESULTS}

Patients' characteristics including disease and treatment information prior to administration of LBT are depicted in Table 1. Thirty eight (57.0\%) patients received adjuvant chemotherapy and 26 of them (68.0\%) received adjuvant $\mathrm{T}$ based chemotherapy. Median disease free survival was $22(0-120)$ months. All except 4 patients received T either in the adjuvant $(n=26)$ or palliative $(n=57)$ settings. Two of these 4 patients presented with stage III disease, refused adjuvant chemotherapy and received adjuvant hormonal therapy. The other 2 patients presented with stage IV disease at initial diagnosis, refused palliative intravenous treatment and received first line palliative hormonal therapy.

$L$ was combined with $C$ in $58(86.6 \%)$ patients while $9(13.4 \%)$ patients received either $L$ in combination with other agents or as a single anti-cancer treatment (see Table 1). In 9/67 (13.4\%) patients LBT was the first line of treatment for A/MHER2 ${ }^{+} B C$. Six of these 9 patients received adjuvant chemotherapy and $\mathrm{T}$ prior to development of advanced disease. The other 3 patients were never exposed to $T$ as they refused intravenous treatment and received first line palliative $L$ combined with $C$, letrozole and exemestane ( 1 in each patient). Dose delay for $>1$ week or discon- tinuation due to toxicity was reported in 13 (19.4\%) and dose reduction in 9 (13.4\%) patients. Sixteen patients $(24.0 \%)$ had dose delay $>1$ week and/or dose reduction.

Table 1. Patients' characteristics

\begin{tabular}{|c|c|}
\hline Characteristics & $\begin{array}{c}\text { Number of pa- } \\
\text { tients, } n(\%)\end{array}$ \\
\hline \multicolumn{2}{|l|}{ Stage at primary diagnosis $(n=67)$ : } \\
\hline I & $1(1.5)$ \\
\hline II & $12(17.9)$ \\
\hline III & $35(52.2)$ \\
\hline IV & $19(28.4)$ \\
\hline \multicolumn{2}{|c|}{ Estrogen and/or progesterone receptors $(n=67)$ : } \\
\hline Positive & $33(49.0)$ \\
\hline Negative & $33(49.0)$ \\
\hline Unknown & $1(2.0)$ \\
\hline \multicolumn{2}{|l|}{ Adjuvant chemotherapy $(n=67)$ : } \\
\hline No & $29(43.0)$ \\
\hline Yes: & $38(57.0)$ \\
\hline $\mathrm{T}$ based & $26(68.0)$ \\
\hline non-T based & $12(32.0)$ \\
\hline Disease free survival & $\begin{array}{c}\text { Median }=22 \\
(0-120) \text { months }\end{array}$ \\
\hline \multicolumn{2}{|l|}{ Lines of palliative chemotherapy prior to $L(n=67)$ : } \\
\hline None & $9(13.4)$ \\
\hline One line & $37(55.2)$ \\
\hline Two lines & $15(22.4)$ \\
\hline Three lines & $6(9.0)$ \\
\hline Palliative chemotherapy prior to $L(n=67)$ : & $58(86.6)$ \\
\hline$T$ based $(n=58)$ & $57(98.0)$ \\
\hline non-T based $(n=58)$ & $1(2.0)$ \\
\hline Age at start of $L$ & $\begin{array}{c}\text { Median }= \\
46(22-70) \text { years }\end{array}$ \\
\hline \multicolumn{2}{|l|}{$L$ based regimens $(n=67)$ : } \\
\hline LC & $58(86.6)$ \\
\hline $\mathrm{L}$ and letrozole & $3(4.5)$ \\
\hline $\mathrm{L}$ and exemestane & $2(3.0)$ \\
\hline Single agent $\mathrm{L}$ & $2(3.0)$ \\
\hline $\mathrm{L}$ and $\mathrm{T}$ & $1(1.5)$ \\
\hline $\mathrm{L}$ and vinorelbine (LV) & $1(1.5)$ \\
\hline
\end{tabular}

3 patients were not evaluable for response. Objective response in evaluable patients was $41 / 64$ (64.0\%) patients in all patients, $36 / 56(64.0 \%)$ in patients who received LC and $5 / 8(62.5 \%)$ in patients who received other LBT regimens. Detailed response to treatment is depicted in Table 2.

Table 2. Response to LBT

\begin{tabular}{lccc}
\hline \multicolumn{1}{c}{ Variables } & All patients, $\mathrm{n}(\%)$ & $\begin{array}{c}\text { LC regimen, } \\
\mathrm{n}(\%)\end{array}$ & $\begin{array}{c}\text { Other regimens, } \\
\mathrm{n}(\%)\end{array}$ \\
\hline $\begin{array}{l}\text { Total number } \\
\text { Evaluable for re- }\end{array}$ & 67 & 58 & 9 \\
sponse & 64 & 56 & 8 \\
CR & $5(8.0)$ & $5(9.0)$ & $0(0.0)$ \\
PR & $36(56.0)$ & $31(55.0)$ & $5(62.5)$ \\
Objective re- & $41(64.0)$ & $36(64.0)$ & $5(62.5)$ \\
sponse & & & \\
(CR + PR) & & & \\
SD & $11(17.0)$ & $10(18.0)$ & $1(12.5)$ \\
PD & $12(19.0)$ & $10(18.0)$ & $2(25.0)$ \\
\hline
\end{tabular}

Note: $\mathrm{CR}$ - complete response; PR - partial response; SD - stable disease; PD - progressive disease.

After a median follow up of 18 (4-38) months, the median PFS was 10 months (95\% Cl: 7.8-12.2) (Fig. 1). Median OS was 27 months (95\% Cl: 12.13-41.87) (Fig. 2).

At time of analysis (October 2013), 26 (39.0\%) patients were still continuing LBT while 41 (61.0\%) stopped this treatment due to progressive disease $(n=36)$, toxicity $(n=2)$ and lost to follow up $(n=3)$. $20(30.0 \%)$ patients received at least one subsequent 
line of chemotherapy yielding objective response in $36.0 \%$ of evaluable patients.

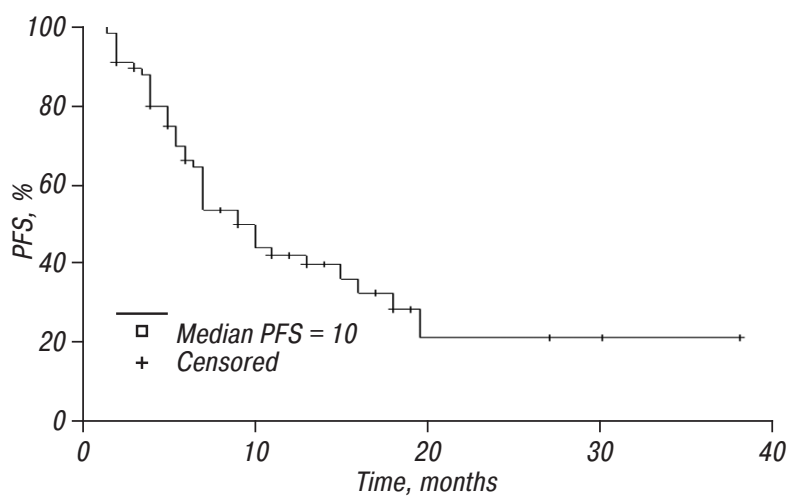

Fig. 1. PFS of all patients $(n=67)$

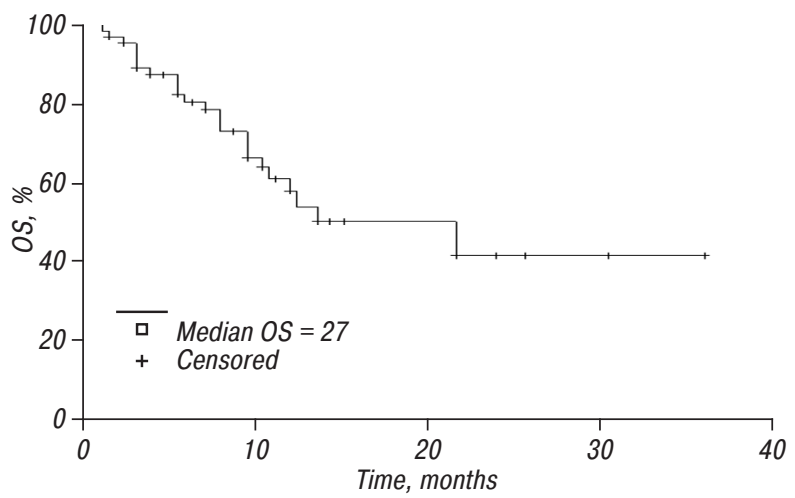

Fig. 2. OS of all patients $(n=67)$

\section{DISCUSSION}

$\mathrm{LC}$ is an established systemic treatment for patients with $\mathrm{A} / \mathrm{MHER} 2^{+} \mathrm{BC}$ who received prior therapy including $T$. The rational for this treatment are the results of the landmark registration phase III that randomized patients to single agent $\mathrm{C}$ or LC. Median time to progression in was 8.4 months in LC group and 4.4 in $\mathrm{C}$ group (HR 0.49; 95\% Cl: 0.34-0.71; $p<0.001$ ) [5].

There are some similarities and differences between our patients and patients included in the registration trial. The differences can be explained by the strict prespecified patients' selection in prospective research setting and the relatively flexible approach in daily real life practice. For example, in the registration trial all patients received prior $\mathrm{T}$ in adjuvant $(5.0 \%)$ or metastatic (95.0\%) settings. However, in our study 4 (6.0\%) patients have never received prior T due to patients' own choice. Another possible reason for differences is variations in social and disease characteristics among both studies population. For example, in registration trial, the median age was 51 and 54 years in each treatment arm and was 46 years in our population. This reflects the Saudi Arabian community young age distribution where only $5 \%$ of the whole population are 60 years or older according to the year 2013 United Nations report [10].

In addition, some reports show that women from the Middle East develop BC at relatively younger age. Age at diagnosis of BC was $<45$ years in $49 \%$ of 6922 women with BC in an epidemiological study from Saudi Arabia [11]. Previous work by our group reported that median age of women with $\mathrm{HER} 2^{+} \mathrm{BC}$ was 45 years [2]. Despite these minor differences, our patients generally represent HER2 ${ }^{+} \mathrm{BC}$ population. For example, $49.0 \%$ of our patients have hormone receptor positive disease which is similar to $48.0 \%$ in the registration trial, $50.0 \%$ in the adjuvant HERA phase III international study and $50 \%$ in a previous work by our group [2-4].

Objective response to LC in our patient was $64.0 \%$. This is higher than what was reported in the registration trial (22.0\%). Possible explanation include (a) assessment was performed by reviewing imaging and/or reports by the treating medical oncologists and not by a radiologist (b) a radiology report may describe a response while tumor shrinkage is less than needed for accurate definition of response and thus overestimate response rates (c) small number of patients in our study. It is well recognized that response rates are usually higher in retrospective and in smaller than larger studies. Response to LC was $34 ; 41 ; 43$ and $44 \%$ in 4 different studies each recruited 26; 116; 68 and 52 patients, respectively [12-15].

It is reassuring that response was also seen in 5 out of 9 patients who received either $L$ in combination with other agents or as a single anti-cancer treatment. Adding $L$ to chemotherapy has proven to improve outcome in clinical trial. For example $L$ improved response rate ( 69 vs. $50 \%$ ), PFS ( 9.7 vs. 6.5 months) and OS ( 27.8 vs. 20.5 months) when added to paclitaxel compared to paclitaxel alone [16]. Similar results were reported in the subset of patients with HER2 overexpressed disease from another trial [17]. Response to combined $L$ and vinorelbine (LV) is reported in 31 and $14 \%$ of patients in phase I and single arm phase II trials $[18,19]$. A phase II study randomized 37 patients to LC and 75 patients - to LV. Response rates were $35 \%$ in LC and $20 \%$ in LV arms. Median PFS was 6.2 in both arms. Median OS was 19.4 months in LC and 24.3 months in LV arms (HR 1.02; 95\% Cl 0.50-2.06). These results indicate that $L V$ offers an effective treatment option that is comparable LC [20].

Adding $L$ the hormonal therapy letrozole has also proven to improve outcome in these patients compared to hormonal therapy alone [21, 22]. Only one of our patients received L combined with T (LT). She achieved partial response and PFS of 7 months.

The prospect of dual HER2 blockage is intriguing form the efficacy point of view but also as a nonchemotherapy combination. A phase III trial randomized patients with $\mathrm{A} / \mathrm{MHER} 2^{+} \mathrm{BC}$ after progression on $T$ to single agent $L$ or LT. Response were achieved in 7 and $10 \%$ and Clinical benefit in 12 and 25\% in $L$ and LT arms, respectively [8]. Updated final analysis confirmed superiority LT in PFS (HR 0.74; $95 \% \mathrm{Cl} 0.58-0.94 ; \mathrm{p}=0.011)$ and in OS benefit (HR 0.74; 95\% Cl 0.57-0.97; $\mathrm{p}=0.026$ ) despite the crossover of $52 \%$ of patients from $L$ arm to receive subsequent $\mathrm{T}$ [23]. Response rate of $8.0 \%$ and clinical benefit rate of $14.1 \%$ were reported in a phase II trial of single agent $L[6]$. Two of our patients received sin- 
gle agent $L$. Both of them achieved objective response. PFS and OS were of 14 and 15 months for one patient and 7 and 10 months of the other. Both did not receive any subsequent anti-cancer therapy. After a median follow up of 18 (4-38) months, the median PFS was 10 months in all patients and 10 months in 58 patients who received LC. This is in line with the PFS duration of 8.4 months reported in the LC arm of the registration trial [5]. Median OS was 27 months in all patients and 17 months in patients who received LC. This is exactly the same median OS of 75 weeks (equivalent to 17 months) reported in LC arm at the updated analysis of the registration trial [24].

In the registration phase III trial, most adverse events in LC arm were of grades I and II. Grade III serious adverse events were diarrhea $(12.0 \%)$ and hand foot syndrome $(7.0 \%)$ while other grade III adverse events were reported in $\leqslant 2 \%$ of patients. Only 2 patients developed grade IV toxicity. Adverse events led to discontinuation of treatment in 22 patients in LC arm [5]. Detailed documentation of toxicity in the context of a clinical study is a predefined and a well-structured exercise allowing capture of all expected and unexpected adverse events and their grades and outcome. However, this is not necessarily the case during daily routine clinical practice. And thus we expected challenges in extracting meaningful data on toxicity. For this reason we used dose delay for $>1$ week (including discontinuation) and dose reduction due to adverse events as surrogates for serious toxicity. Dose delay was required in $13(19.0 \%)$ and dose reduction in $9(13.0 \%)$ patients. $16(24.0 \%)$ patients had dose delay $>1$ week and/or dose reduction. $6(9.0 \%)$ patients required both dose reduction and dose delay $>1$ week (including 2 regimen discontinuations) due to toxicity. According to these findings and allowing for the limitations in the way we explored toxicity, tolerance to LBT in our patients is generally in line with what is expected and with the registration trial data.

Our patients are generally heavily pretreated. Most of them received hormonal therapy when appropriate, adjuvant chemotherapy, palliative chemotherapy (1-3 lines) and $T$ in either adjuvant and/or advanced settings. Despite heavy pretreatment and after failure of LBT, 20 (30.0\%) patients received a subsequent line of chemotherapy (including $L$ based in 7 patients). 14 of them were evaluable for response. $5 / 14$ (36.0\%) achieved objective response. 7 (10.0\%) patients received a second subsequent line of chemotherapy (including $L$ based in 1 patient). This observation indicates that LBT does not limit feasibility of subsequent chemotherapy and its benefit.

\section{CONCLUSION}

In daily clinical practice, $\mathrm{L}$ is mostly combined with $\mathrm{C}$ for the treatment of women with $\mathrm{A} / \mathrm{MHER} 2^{+} \mathrm{BC}$ after progression on $\mathrm{T}$. However, other $\mathrm{L}$ based combinations are feasible and effective. In this setting, LBT produces meaningful tumor responses, PFS and OS are comparable to (and may exceed) those reported in clinical trials. This benefit is likely to add to the survival gain achieved by prior and subsequent lines of therapy.

\section{REFERENCES}

1. Slamon DJ, Clark GM, Wong SG, et al. Human breast cancer: correlation of relapse and survival with amplification of the HER-2/neu oncogene. Science 1987; 235: 177-82.

2. Zekri JM, Ibrahim E, Sadiq BB, et al. A matched group study of triple negative versus HER-2 positive (irrespective of hormonal status) breast cancer: two subtypes with highrisk features and poor outcome. Ecancermedicalscience 2010; 4: 167. doi: 10.3332/ecancer.2010.167.

3. Piccart-Gebhart MJ, Procter M, Leyland-Jones B, et al. Trastuzumab after adjuvant chemotherapy in HER2-positive breast cancer. N Engl J Med 2005; 353: 1659-72.

4. Slamon DJ, Leyland-Jones B, Shak S, et al. Use of chemotherapy plus a monoclonal antibody against HER2 for metastatic breast cancer that overexpresses HER2. N Engl J Med 2001; 344: 783-92.

5. Geyer CE, Forster J, Lindquist D, et al. Lapatinib plus capecitabine for HER2-positive advanced breast cancer. N Engl J Med 2006; 355: 2733-43.

6. Blackwell KL, Pegram MD, Tan-Chiu E, et al. Singleagent lapatinib for HER2-overexpressing advanced or metastatic breast cancer that progressed on first- or second-line trastuzumab-containing regimens. Ann Oncol 2009; 20: 1026-31.

7. Fleeman N, Bagust A, Boland A, et al. Lapatinib and trastuzumab in combination with an aromatase inhibitor for the first-line treatment of metastatic hormone receptor-positive breast cancer which over-expresses human epidermal growth factor 2 (HER2): a systematic review and economic analysis. Health Technology Assessment 2011; 15: 2011. DOI: 10.3310/hta15420.

8. Blackwell KL, Burstein HJ, Storniolo AM, et al. Randomized study of lapatinib alone or in combination with trastuzumab in women with ErbB2-positive, trastuzumab-refractory metastatic breast cancer. J Clin Oncol 2010; 28: 1124-30.

9. Botrel TE, Paladini L, Clark OA. Lapatinib plus chemotherapy or endocrine therapy (CET) versus CET alone in the treatment of HER-2-overexpressing locally advanced or metastatic breast cancer: systematic review and metaanalysis. Core Evid 2013; 8: 69-78.

10. Population Indicators: World Population Policies. 2013. New York: United Nations, 2013: http://www.un.org/en/development/ desa/population/publications/pdf/policy/WPP2013/Country_Profiles/SaudiArabia_Demographic.pdf.

11. Alghamdi IG, Hussain II, Alghamdi MS, ElSheemy MA. The incidence rate of female breast cancer in Saudi Arabia: an observational descriptive epidemiological analysis of data from Saudi Cancer Registry 2001-2008. Breast Cancer 2013; 5: 103-9.

12. Cetin B, Benekli M, Dane F, et al. Lapatinib plus capecitabine for HER2-positive advanced-stage breast cancer in elderly women: review of the Anatolian Society of Medical Oncology (ASMO) experience. Breast Care 2013; 8: 67-70.

13. Martin M, Bonneterre J, Geyer ChE, et al. A phase two randomised trial of neratinib monotherapy versus lapatinib plus capecitabine combination therapy in patients with HER2+ advanced breast cancer. Eur J Cancer 2013; 49: 3763-72.

14. Crivellari D, Spazzapan S, Lombardi D, et al. Lapatinibbased therapy in heavily pretreated HER2-positive metastatic breast cancer: a single institution experience. Tumori 2012; 98: 33-8.

15. Xu B-H, Jiang Z-F, Chua D, et al. Lapatinib plus capecitabine in treating HER2-positive advanced breast cancer: efficacy, safety, and biomarker results from Chinese patients. Chin J Cancer 2011; 30: 327-35. 
16. Guan Z, Xu B, DeSilvio ML, et al. Randomized trial of lapatinib versus placebo added to paclitaxel in the treatment of human epidermal growth factor receptor 2-overexpressing metastatic breast cancer. J Clin Oncol 2013; 31: 1947-53.

17. Di Leo A, Gomez HL, Aziz Z, et al. Phase III, doubleblind, randomized study comparing lapatinib plus paclitaxel with placebo plus paclitaxel as first-line treatment for metastatic breast cancer. J Clin Oncol 2008; 26: 5544-52.

18. Brain E, Isambert N, Dalenc F, et al. Phase I study of lapatinib plus vinorelbine in patients with locally advanced or metastatic breast cancer overexpressing HER2. Br J Cancer 2012; 106: 673-7.

19. Saip P, Eralp Y, Sen F, et al. Phase II study of lapatinib in combination with vinorelbine in patients with HER2 positive recurrent or metastatic breast cancer: a multicentric Turkish Oncology Group (TOG) trial. Breast 2013; 22: 628-33.

20. Janni W, Sarosiek T, Karaszewska B, et al. A phase II, randomized, multicenter study evaluating the combination of lapatinib and vinorelbine in women with ErbB2 overex- pressing metastatic breast cancer. Breast Cancer Res Treat 2014; 143: 493-505.

21. Johnston S, Pippen J Jr, Pivot X, et al. Lapatinib combined with letrozole versus letrozole and placebo as firstline therapy for postmenopausal hormone receptor-positive metastatic breast cancer. J Clin Oncol 2009; 27: 5538-46.

22. Schwartzberg LS, Franco SX, Florance A, et al. Lapatinib plus letrozole as first-line therapy for HER-2+ hormone receptor-positive metastatic breast cancer. Oncologist 2010; 15: 122-9.

23. Blackwell KL, Burstein HJ, Storniolo AM, et al. Overall survival benefit with lapatinib in combination with trastuzumab for patients with human epidermal growth factor receptor 2-positive metastatic breast cancer: final results from the EGF 104900 Study. J Clin Oncol 2012; 30: 2585-92.

24. Cameron D, Casey M, Oliva C, et al. Lapatinib plus capecitabine in women with HER-2-positive advanced breast cancer: final survival analysis of a phase III randomized trial. Oncologist 2010; 15: 924-34. 